\title{
Radiation Dose Image Quality Optomization in Dental Implantalogy
}

\author{
Ahmed Al-Humairi ${ }^{1}$, Xiaoming Zheng ${ }^{1}$, Ho Leung Ip $^{1}$ and Bilal El Masoud ${ }^{2,3}$ \\ ${ }^{1}$ Charles Sturt University, Wagga Wagga, NSW, Australia. \\ ${ }^{2}$ Charles Sturt University, Orange, NSW, Australia. \\ ${ }^{3}$ Jordan University of Science and Technology, Irbid. Jordan
}

\begin{abstract}
This paper is aimed to investigate the possibility of using a new method of dose and image quality optimization by employing visual grading analysis with a slight modification. Cone beam CT was utilized with different exposure settings to acquisition several images to skull phantom. Three different observers were involved with moderate agreement using Kappa factor. As a result, the new method introduced could be applied successfully in radiation dose and image quality optimization with the potential of achieving low dose level protocols with acceptable image quality in dental implantalogy.
\end{abstract}

Keywords-optimization; image quality, CBCT; visual grading analysis

\section{INTRODUCTION}

Today, oral implants are utilized routinely in the oral rehabilitation with great success rates. This requires adequate planning, which is suggested to be one of the main vital aspects in establishing implant success. However, bone perforation, neurosensory disturbances and hemorrhage complications have been evident in the frequent placement of oral implants [1]. Thus, it is crucial to acquire accurate information regarding anatomical vital structures preceding placement of implants [2]. The location of anatomical structures and topography are visualized through radiographic images. Several imaging options have been suggested for implant treatment planning; such as intra-oral radiography, conventional extra-oral radiography, tomography, computed tomography (CT) and cone beam computed tomography (CBCT) $[3,4,5]$.

Some of the most significant radiological diagnostic procedures in dentistry are the utilization of the currently firmly established 3D imaging methods. The cone beam CTs have been broadly instigated in the clinical dental practice with diverse applications resulting in the patient receiving high radiation doses compared to earlier diagnostic techniques. Despite the widespread use of cone beam CT, concerns regarding radiation dose and risks associated with the patient as well as the general population have been addressed [6].

Evaluation methods of image quality play a vital role of CBCT imaging optimization, although it is not well outlined for its clinical application in relation to human observer. Published CBCT studies that focus on clinical evaluation of image quality have mostly been based on subjective analysis, i.e. visualization of anatomical landmarks [7], utilizing various indexes such as visual grading analysis (VGA) which is an incredibly conventional tool in implant planning. However, this system relies on average evaluation scores of observer numbers, and due to that, the VGA values are ordinal in nature and thus scrutiny of these data need to be vigilantly considered [8].

Hence to overcome the ordinal nature of the VGA, other procedures including visual grading characteristics (VGC) and ordinal regression systems have been applied in terms of visual grading analysis of the image quality. Within the frame of employing the VGA data, the VGC methodology is reflection to the ROC studies [8]. Nevertheless, there is no substantial application of visual grading characteristics because of the complexity to acquire large number of data within exposure settings of $\mathrm{CR}$ and $\mathrm{CBCT}$ in dentistry, in addition to the fluctuant observer reliability. To this current day, not one defined method has been concluded for radiation dose optimization with respect to cone beam CT in dental implantology.

The magnitude of the in-vitro study is provoking the dose reduction protocols by utilizing phantoms to investigate the link between the acceptable image quality and dose reduction. This connection will potentially be implicated in the clinical field [9]. Moreover, Rivas et al., 2015 [10] had presented a simple system of VGA application to create low-dose protocol for CBCT in children, where it is founded on observer agreement of satisfactory image quality. In this current study, the objective is to analyze the image quality of the mandible pre-surgical implant planning by employing varying exposure parameters of cone beam computed tomography. The images would be assessed based on visual grading analysis without the use of reference images; modified methodology to Rivas et al., 2015 will be employed.

\section{MATERIAL AND METHODS}

A skull phantom; a dry adult human skull entrenched in plexi-simulating the soft tissue was used in this study. The skull was imaged with a cone beam computed tomography (Planmeca Promax 3D Max) operated at 70, 80 and $96 \mathrm{kVp}$ and several tube currents $(4,6,8,10$, and $12 \mathrm{~mA})$ in full field of view to the maxillofacial area .

Ethics and radiation safety committee approval were obtained prior to undertaking the experiment from Charles Sturt University (ethic committee and radiation safety board). The radiation doses were recorded as a dose area product (DAP). 
All imaging studies were reviewed in blinded random order and independently by three specialists experienced in dental implants ( $\mathrm{ZH}, \mathrm{BE}$, and $\mathrm{SC})$. The observers were in charge of evaluating the visibility of anatomical landmarks quality and overall image quality pre-surgical of implant placement. The observers ranked 7 anatomical landmarks questions (ALQ) on a 5 point rating scale; 1 : definitely it is not clearly visible, 2 : probably it is not clearly visible, 3 : indecisive whether that is visible or not, 4: probably it is clearly visible and 5: definitely it is clearly visible. In addition, an overall image quality (OIQ) regarding pre-surgical implant placement on 3 point rating scale (poor, acceptable and clear) was recorded.

The image analysis was based on the requirement of the image pre-surgical stage of implant placement in the mandible; left-side of the mandible was assessed. In each image 3 or 4 views has been presented. Therefore, the assessment of each ALO required interpretation of the two different views. The score of all observers were average for each observer at every exposure setting. The need of the clinician to examine the site prior to implant placement was the purpose of all images required to be assessed. Furthermore, the observers demonstrate overall analysis of the images and whether the images are acceptable for their diagnostic task for pre-surgical implant placement site.

A low light control area was utilized to evaluate all images. Observers were instructed to have their eyes rested if they felt their eyes strain. In addition, observers were required to replicate the clinical situation such as wearing their glasses and using any magnification methods if they clinically use them. Intra-observer and inter-observer rating were assessed.

\section{RESULTS}

The results of the subjective method of evaluation of the image quality were reported in this study as mentioned in Table1. The image quality method was based on specific criteria as outlined below:

\section{Denote by $\boldsymbol{X}_{\boldsymbol{f}}$ the score of the $\boldsymbol{f}$-the ALQ given by the $i$-the observer and $N_{l}=\sum Z_{i=1} 1\left\{X_{U} \geq 4\right\}$ where $1\{A\}$ is an indicater function which takes a value of 1 if the condition $A$ is met and $D$ otherwise. In other words, $\mathrm{N}_{l}$ is the number of the seven ALQ scored 4 or 5 for Observer $t$. Define}

$$
C=\sum_{i=1}^{3} 1\left\{\mu_{i} \geq 5 \cap Q_{i} \geq 2\right\},
$$

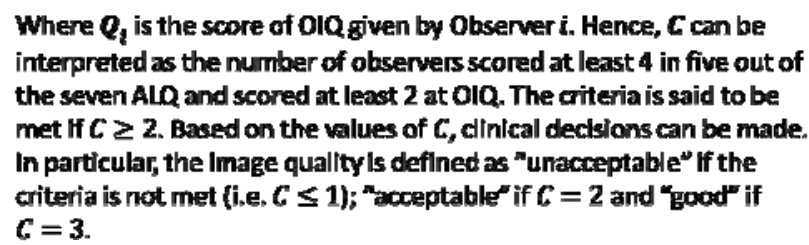

FIGURE I. IMAGE QUALITY CRITERIA
TABLE I. IMAGE QUALITY IN THE FORM OF VGA BY THE OBSERVERS, WHERE "U" STANDS FOR "UNACCEPTABLE", "A" STANDS FOR "ACCEPTABLE", "G" STANDS FOR "GOOD", "N" STANDS FOR THE NUMBER OF THE SEVEN ALQ SCORED 4 OR 5 FOR OBSERVER AND "Q" IS THE SCORE OF OIQ GIVEN BY OBSERVER

\begin{tabular}{|c|c|c|c|c|c|c|c|c|c|}
\hline $\begin{array}{c}\text { Exposure } \\
\text { settings }\end{array}$ & \multicolumn{2}{c|}{$\begin{array}{c}\text { Observer } \\
\mathbf{1}\end{array}$} & \multicolumn{2}{c|}{ Observer 2 } & \multicolumn{2}{c|}{$\begin{array}{c}\text { Observer } \\
\mathbf{3}\end{array}$} & $\begin{array}{c}\text { Criteria } \\
\text { met?b }\end{array}$ & $\begin{array}{c}\text { Clinical } \\
\text { Decision }\end{array}$ \\
\hline $\mathrm{kVp}$ & $\mathrm{mA}$ & $\mathrm{N}$ & $\mathrm{Q}$ & $\mathrm{N}$ & $\mathrm{Q}$ & $\mathrm{N}$ & $\mathrm{Q}$ & & \\
\hline 70 & 8 & 2 & 1 & 1 & 1 & 6 & 2 & No & $\mathrm{U}$ \\
\hline 70 & 10 & 4 & 1 & 2 & 1 & 5 & 1 & No & $\mathrm{U}$ \\
\hline 70 & 12 & 2 & 1 & 1 & 1 & 5 & 1 & No & $\mathrm{U}$ \\
\hline 80 & 4 & 2 & 1 & 5 & 1 & 7 & 2 & No & $\mathrm{U}$ \\
\hline 80 & 6 & 4 & 1 & 7 & 2 & 7 & 2 & Yes & $\mathrm{A}$ \\
\hline 80 & 8 & 6 & 2 & 7 & 2 & 6 & 3 & Yes & $\mathrm{G}$ \\
\hline 80 & 10 & 7 & 2 & 6 & 2 & 7 & 3 & Yes & $\mathrm{G}$ \\
\hline 80 & 12 & 3 & 1 & 6 & 2 & 7 & 3 & Yes & $\mathrm{A}$ \\
\hline 96 & 4 & 5 & 1 & 5 & 2 & 6 & 2 & Yes & $\mathrm{A}$ \\
\hline 96 & 6 & 6 & 2 & 7 & 2 & 6 & 2 & Yes & $\mathrm{G}$ \\
\hline 96 & 8 & 7 & 2 & 5 & 1 & 6 & 2 & Yes & $\mathrm{A}$ \\
\hline 96 & 10 & 2 & 1 & 7 & 3 & 6 & 2 & Yes & $\mathrm{A}$ \\
\hline 96 & 12 & 4 & 1 & 7 & 2 & 7 & 2 & Yes & $\mathrm{A}$ \\
\hline
\end{tabular}

TABLE II. EXTRACTED FROM RIVAS ET AL. (2015, BJR, TABLE 3, P. 10) WITH SOME MODIFICATION, WHERE "U" STANDS FOR "UNACCEPTABLE", "A" STANDS FOR "ACCEPTABLE", "G" STANDS FOR "GOOD". THE THICK LINE INDICATES THE THRESHOLD COMBINATIONS OF KVP AND MA WHICH THE OVERALL QUALITY IS AT LEAST ACCEPTABLE. THE NUMBER IN THE BRACKET IS THE DAP

\begin{tabular}{|c|c|c|c|c|c|c|}
\hline \multirow{2}{*}{\multicolumn{2}{|c|}{ CBCT }} & \multicolumn{5}{|c|}{$m A$} \\
\hline & & \multirow[t]{2}{*}{4} & \multirow[t]{2}{*}{6} & \multirow{2}{*}{$\begin{array}{c}8 \\
\\
U \\
(434)\end{array}$} & \multirow{2}{*}{$\begin{array}{c}10 \\
U(548)\end{array}$} & \multirow{2}{*}{$\begin{array}{c}12 \\
U \\
(659)\end{array}$} \\
\hline \multirow{3}{*}{$k V P$} & 70 & & & & & \\
\hline & 80 & $\mathrm{U}(359)$ & $\begin{array}{c}\mathrm{A} \\
(539)\end{array}$ & $\begin{array}{c}\mathrm{G} \\
(719)\end{array}$ & G (898) & $\begin{array}{c}\text { A } \\
(1078)\end{array}$ \\
\hline & 96 & A (654) & $\begin{array}{c}\text { G } \\
(981)\end{array}$ & $\begin{array}{c}\text { A } \\
(1308)\end{array}$ & A (1635) & $\begin{array}{c}\text { A } \\
(1962)\end{array}$ \\
\hline
\end{tabular}

This result has recognized a threshold in regards to exposure setting sequences of the radiation dose which offered acceptable-good image quality. The minimum acceptable exposure setting is $\mathrm{kVP}=80$ and $\mathrm{mA}=6$ with Dose area product (DAP) $\mathrm{mGy} \mathrm{cm}^{2} 539$. However, the exposure settings $\mathrm{kVP}=80$,

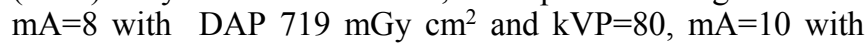

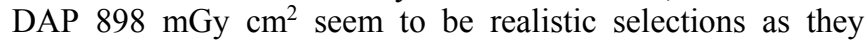
attain good clinical image quality, which is more enhanced than the minimum acceptable combination.

Taking this into consideration, the clinical acquisition quality of CBCT may be manipulated by other dynamics such as patient positioning and metal artifact movement (Table 2). Intra- and Inter-observer agreements were calculated using Kappa factor [11] and for each individual question. 
Moderate inter-observer agreements were established as mentioned in Table 3. The observer blindly evaluated the images where some of the images were repeated intentionally to evaluate the intra-observer agreement. With the intraobserver agreement, observer moderate agreements were evaluated with Kappa value $\geq 0.4$ (Table 3 ). Based on previous studies if the Kappa value presented as less than 0.4; the observer should be eliminated from the study $[12,13]$.

TABLE III. INTER AND INTRA-OBSERVER AGREEMENT FOR THREE OBSERVERS USING KAPPA AGREEMENT

\begin{tabular}{|c|c|c|c|}
\hline \multicolumn{5}{|c|}{ Inter-Observer Reliability: } \\
\hline Observer & 1 & 2 & 3 \\
\hline 1 & NA & 0.322 & 0.261 \\
\hline 2 & & NA & 0.468 \\
\hline 3 & \multicolumn{4}{|c|}{ Intra-Observer Reliability: } \\
\hline Observer 1 & Observer 2 & Observer 3 \\
\hline 0.477 & 0.436 & 0.777 & \\
\hline
\end{tabular}

\section{DISCUSSION}

All clinicians involved in the clinical management especially for implantology must be able to apply what they can interpret from the image to the clinical case. The actual observation needs of each clinician prior to implant placement must be taken into consideration, as occasionally two dimension images can be regarded as enough for planning [2] .

On the other hand, this study was performed to test the new optimization method by Rivas et al., The conception of this research is to initiate a new optimization technique in regards to $\mathrm{CBCT}$ in implant dentistry[14,15]. Numerous researchers have assessed the image quality and radiation dose without generating a conclusive standardized low dose protocol. Harris et al, posed a general principle rather than a specific one in regards to exposure settings [16].

Other studies have analyzed the image quality for different CBCT equipment and estimated the dose with such equipment without a specific link between them to construct the most acceptable image quality with the lowest dose.

This study is only valid for this CBCT equipment, and is suggested to be used for several CBCT apparatus to fabricate a protocol regarding each clinical indication where sequence is deemed a good starting point for DRLs (Dose reference level) with respect to CBCT in Australia.

In past research, the five score measurements by observers with respect to anatomical landmarks have been widely utilized. Though, these image quality assessment scores have not been applied to generate a low dose protocol, but instead to compare between equipment and different clinical indications [17] .

The results are in agreement with the main principle research by Rivas et al, with the prospect of decreasing the dose with respect to acceptable image quality. This brings into perspective similar agreements to the study by other study [18], where physical image evaluation apparatus was employed.
As a summary the dose reduction can be approximately $50 \%$ of the used one, which stands parallel to other previous studies. One of the drawbacks in this study was the low inter and intra reliability agreement between the observers, and it has also been reported in Rivas et al., This can be overcome by having more observers to take part in the evaluation where it can lead to reducing the disagreement gap between the observers.

Furthermore, low exposure setting variety in this study as compared to previous studies has been observed. This study is considered as preliminary for a broader framework in the application of the image quality evaluation and optimization technique in dental radiology, with particular interest; implantology.

\section{CONCLUSION}

New technique with respect to dose optimization has successful applications in dental radiology, generally in DRL and particularly in implantalogy. This result must be regarded with caution; it may have several other limitations such as presence of different CBCT apparatus, different clinical protocols and the agreement between the observers.

\section{REFERENCES}

[1] G. Greenstein, J. Cavallaro, G. Romanos, and D. Tarnow. "Clinical recommendations for avoiding and managing surgical complications associated with implant dentistry: A review". J Periodontol 2008;79:1317-1329.

[2] H. L. Chan, K. Misch, and H.L. Wang. "Dental imaging in implant treatment planning". Imlant dentistry 2010; vol 19; 4:288-297.

[3] T. Dreiseidler, R. Mischkowski, J. Neugebauer, L. Ritter and J. E. Zöller. "Comparison of cone-beam imaging with orthopantomography and computerized Tomography for assessment in presurgical implant dentistry". Intj Oral Maxillofac Implants 2009;24:216-225.

[4] L. Pertl, B. Gashi-Cenkoglu, J. Reichmann, N. Jakse and C Pertl. "Preoperative assessment of the mandibular canal in implant surgery: comparison of rotational panoramic radiography (OPG), computed tomography (CT) and cone beam computed tomography (CBCT) for preoperative assessment in implant surgery". Eur J Oral Implantol 2013;6(1):73-80.

[5] F. Dammann, F. Bootz, M. Cohnen, S. Haßfeld, M. Tatagiba and S. Kösling. "Diagnostic imaging modalities in head and neck disease". Dtsch Arztebl Int 2014; 111: 417-23. DOI: 10.3238/arztebl.2014.0417.

[6] W. C. Scarfe and A. G. Farman, P. Sukovic. "Clinical applications of cone-beam computed tomography in dental practice". Can Dent Assoc 2006; 72(1):75-80.

[7] M. Heiland, D. Schulze, G. Adam and R. Schmelzle. "3D-imaging of the facial skeleton with isocentric mobile C-arm system (Siremobil IsoC3D)". Dentomaxillofac Radiol 2003; 32: 21-25. 10.

[8] X. Zheng, M. Kim and S. Yang. "Optimal kVp in chest computed radiography using visual grading score: a comparison between visual grading characteristic and ordinal regression analysis". Proc. SPIE 9783, Medical Imaging 2016: Physics of Medical Imaging, 97836A (March 31, 2016); doi:10.1117/12.2217414.

[9] J. B. Ludlow and M. Ivanovic. "Comparative dosimetry of dental CBCT devices and 64-slice CT for oral and maxillofacial radiology". OOOOE J, 2008, Volume 106, Issue 1, Pages 106-114.

[10] J. A. H. Rivas, K. Horner, B. Thiruvenkatachari, J. Davies, and C. Theodorakou, "Development of a low-dose protocol for cone beam CT examinations of the anterior maxilla in children". Br J Radiol 2015; $88: 20150559$.

[11] D.G. Altman. "Practical statistics for medical research. London, UK: Chapman and Hall/ CRC Texts in Statistical Science; 1990". 
[12] B.C. Meyer, T.M. Hemmen, C.M. Jackson and P.D. Lyden. "Modified national institutes stroke scale for use in stroke clinical trials".Stroke2002;33: 1261-6. doi:10.1161/01. STR.0000015625.87603.A7

[13] P. Armitage, G Berry and J.N.S. Matthews. "Statistical methods in medical research Oxford, UK: Blackwell Science Ltd; 2008".

[14] E. Fanucci, L. Fiaschetti, M. Otria, M. Mataloni, V. Acampora, R. Lione, A. Barlattani and G. Simonetti. "Comparison of different dose reduction system in computed tomography for orthodontic applications". Oral implantalogy J, (2011), N1-2; 14-22.

[15] C. Steiding, D. Kolditz and W. Kalender. "Comparison of methods for acceptance and constancy testing in dental cone-beam computed tomography". Fortschr Röntgenstr 2015; 187(04): 283-290.

[16] D. Harris, K. Horner, K. Grondahl, R. Jacobs, E. Helmrot, G.I.,Benic et al. "E.A.O. guidelines for the use of diagnostic imaging in implant dentistry 2011. A consensus workshop organized by the European Association for Osseointegration at the Medical University of Warsaw." Clin Oral Implants Res 2012; 23, 1243-53.

[17] E. Hofmann, _M. Schmid, M. Sedlmair, R. Banckwitz, U. Hirschfelder and M. Lell. "Comparative study of image quality and radiation dose of cone beam and low-dose multislice computed tomography - an in-vitro investigation". Clinical Oral Investigations, January 2014, Volume 18, Issue 1, pp 301-311.

[18] R. Pauwels , H. Stamatakis, G. Manousaridis, A. Walker, K. Michielsen, H. Bosmans, R. Bogaerts, R. Jacobs, K. Horner and K. Tsiklakis, "The SEDENTEXCT Project Consortium. Development and applicability of a quality control phantom for dental cone-beam CT'. Journal of Applied Clinical Medical Physics, Vol. 12 , No. 4, Fall 2011 